\section{Anomalous Values of Lattice Spacings obtained by Electron Diffraction}

Several observers have recently reported discrepancies in the lattice spacings calculated from the ring or spot patterns given by electron diffraction. When the thin film traversed by electrons ( $30 \mathrm{k.v}$. contains two crystalline substances, the ratios of the spacings are not always in accord with those given by $\mathrm{X}$-ray diffraction. The variations are of the order of 1 per cent, and are far greater than can be accounted for by experimental error.

G. I. Finch and S. Fordham ${ }^{1}$ have shown that this is so in the case of the alkali halides referred to gold as standard, and Dr. E. R. Cooper and $I^{2}$ obtained discrepancies in the case of two metals aluminium and bismuth, and in metal-metal oxide patterns, $\mathrm{Bi}-\mathrm{Al}_{2} \mathrm{O}_{3}, \mathrm{Zn}-\mathrm{ZnO}, \mathrm{Cd}-\mathrm{CdO}$. Finch and Fordham find that $a$ for $\mathrm{NaCl}$ (assuming the $\mathrm{X}$-ray constants for gold) $=\mathbf{5 . 6 6 6} \mathrm{A}$., using $\mathrm{NaCl}$ films 'flashed' in vacuo ( $a$ for $\mathrm{NaCl}(\mathrm{X}$-ray) $=5 \cdot 628 \mathrm{~A}$.).

V. E. Cosslett ${ }^{3}$, using films formed by evaporation of a dilute salt solution, has reported variations of $a$ with order of reflection, which he suggests are due to refraction or surface lattice effects.

J. Gnan", in a measurement of $h / m$, has calibrated $\mathrm{NaCl}$ against $\mathrm{Bi}$, using a thin collodion film on which $\mathrm{NaCl}$ has been evaporated on one side and $\mathrm{Bi}$ on the other, in a high vacuum. He finds $a$ for $\mathrm{NaCl}(\mathrm{Bi})=$ $5 \cdot 640 \pm 003 \mathrm{~A}$. I found, however, also using evaporated films of chemically pure $\mathrm{NaCl}$ and $\mathrm{Bi}$, that $a$ for $\mathrm{NaCl}(\mathrm{Bi})=5 \cdot 668 \pm \cdot 005 \mathrm{~A}$. Photographs with different exposures gave the same value for $a$, which does not suggest any charging-up effects or heating effects due to inelastic electronic collisions. The constancy of $d_{h k l} \sin \theta$ and the sharpness of the rings appear to preclude effects of refraction or of lattice distortion, and further, the small half-breadth indicates comparatively large crystallites of $\mathrm{NaCl}$ and $\mathrm{Bi}$.

Although slight variations in the $\mathrm{NaCl}$ lattice spacing have been found by X-ray work ${ }^{5}$, these are of the order of 0.01 per cent, and so much smaller than the discrepancies under consideration.

At present, the most reasonable explanation of these anomalies seems to be that of variation of lattice dimensions with crystal size for small erystals, as emphasised by Finch ${ }^{1}$. The use of films evaporated in vacuo, the normal relative intensities of the rings, and the absence of extra rings or bands makes improbable any lattice expansion due to gas absorption. On the other hand, the binding in $\mathrm{Bi}$ is of a homopolar nature ${ }^{6}$ (valency link binding), whilst the $\mathrm{NaCl}$ lattice is governed mainly by ionic forces, so that, according to the simple theory of LennardJones, $a$ for $\mathrm{NaCl}(\mathrm{Bi})$ should be less than $a$ for $\mathrm{NaCl}$ (X-rays), whereas experimentally the reverse is found.

These anomalies may invalidate the results of electron-wave methods for measuring atomic constants, as in the case of Gnan and possibly of S. v. Friesen?, the latter's results depending on the cross grating pattern obtained by transmission of electrons through the very thin fragments projecting on the surface of an etched crystal. Whatever the origin of the discrepancies we have been discussing, their existence is amply proved, and measurements by electron diffraction can scarcely yet be trusted to give accurate relations between the fundamental physical constants.
It is hoped to obtain more definite evidence by using films of bismuth evaporated on to a cleavage flake of mica, since the mica spacings should be reliable, and the $\mathrm{Bi}$ rings can be compared with the spot $(N)$ pattern given by the mica.

\section{E. Pickup.}

Physical Laboratory,

University, Manchester. April 30.

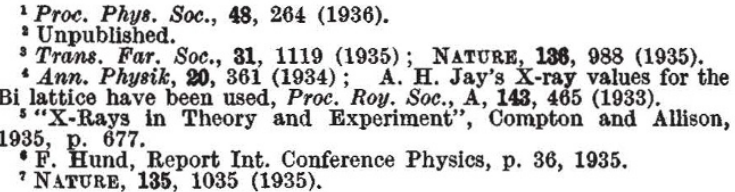

\section{Propagation of Electromagnetic Waves}

IN view of the interpretation of measurements concerning the reflection of radio waves by the ionosphere, it is desirable to calculate the reflected amplitude for a special case, making certain suppositions about the electric properties of the ionosphere and assuming an external magnetic field. I have therefore investigated the propagation of electromagnetic waves in a vertical direction in a medium with electric properties varying in the same direction, and subject to the influence of a constant magnetic field $H$ making an angle $\Theta$ with the vertical.

Studying the case of free ions in a gas (ionosphere) and in an alternating electric field, we can obtain a formula for the mean ionic current density and we can deduce an expression for the dielectric constant $\varepsilon$ and the conductivity constant $g$. Certain assumptions were made by G. J. Elias as to the mean free path of an electron and as to the temperature, and he obtained $^{1}$, for the case of ionisation caused by ultraviolet radiation, the following expressions for $\varepsilon$ and $g$, being functions of the height above the earth :

$$
\varepsilon=1-\eta e^{k z}, \quad g=\frac{\omega}{4 \pi} e^{k z},
$$

where $z=0$ is a fixed plane in the ionosphere, and $\eta$ and $k$ are constants, depending on the physical properties and the condition of the medium.

Owing to the presence of a constant magnetic field, the relations between the ionic current and the electric force components are given by a tensor (as is the case in any anisotropic medium). Using Elias's assumptions, the components of this tensor $\tau$ have been calculated.

A system of axes was introduced, the positive $z$-axis being in the vertical direction, the $y$-axis lying in the $H-z$ plane and the $x$-axis perpendicular.

With the components of $\tau$ and putting $\mu=1$, I derived from the Maxwell equations two simultaneous equations of the second order for $E_{x}$ and $E_{y}$. These equations can be connected in such a way that two ordinary equations for $E_{x} \pm i E_{y}$ are obtained.

In obtaining this result, I neglected some terms; this being permissible for sufficiently short waves and for a sufficiently small value of $H$. Strictly, the reflected waves are elliptically, when the incident ones are circularly, polarised.

So we have one wave, characterised by

$$
\begin{aligned}
& E_{x}+i E_{y} \neq 0 \quad \text { (+wave) } \\
& E_{x}-i E_{y}=0 ;
\end{aligned}
$$

